\title{
Prostanoid receptor genes confer poor prognosis in head and neck squamous cell carcinoma via epigenetic inactivation
}

\author{
Kiyoshi Misawa* (1), Masato Mima, Yamada Satoshi, Atsushi Imai, Daiki Mochizuki, Ryuji Ishikawa, Junya Kita, \\ Yuki Yamaguchi, Shiori Endo, Yuki Misawa and Hiroyuki Mineta
}

\begin{abstract}
Background: Chronic inflammation is a risk factor for head and neck squamous cell carcinoma (HNSCC) and other diseases. Prostanoid receptors are clearly involved in the development of many types of cancer. However, their role is not simple and is poorly understood in HNSCC.

Methods: Methylation profiles of prostanoid receptor family genes were generated for tumour samples obtained from 274 patients with HNSCC, including 69 hypopharynx, 51 larynx, 79 oral cavity, and 75 oropharynx tumour samples, by quantitative methylation-specific PCR. Promoter methylation was then evaluated with respect to various clinical characteristics and patient survival.

Results: The mean number of methylated genes per sample was $2.05 \pm 2.59$ (range 0 to 9). Promoters of PTGDR1, PTGDR2, PTGER1, PTGER2, PTGER3, PTGER4, PTGFR, PTGIR, and TBXA2R were methylated in 43.8\%, 18.2\%, 25.5\%, 17.5\%, $41.2 \%, 8.0 \%, 19.3 \%, 20.4 \%$, and $11.3 \%$ of the samples, respectively. Methylation indices for prostanoid receptor family genes tended to be higher as the number of TET methylation events increased. Patients with 5-9 methylated genes had a significantly lower survival rate than that of patients with 0-4 methylated genes (log-rank test, $P=0.007$ ). In multivariate analyses, PTGDR1 methylation was most highly correlated with recurrence in patients with hypopharyngeal cancer $(P=0.014)$. A similar correlation was observed for PTGER4 in patients with laryngeal cancer $(P=0.046)$. Methylation of the PTGIR and TBXA2R promoters was positively correlated with recurrence in oropharyngeal cancer $(P=0.028$ and $P=0.006$, respectively). Moreover, Patients with 5-9 methylated genes were extremely lower of $5 \mathrm{hmC}$ levels $(P=0.035)$ and was correlated with increasing expression of DNMT3A and DNMT3B ( $\mathrm{P}<0.05$ and $\mathrm{P}<0.05$, respectively).
\end{abstract}

Conclusion: We characterised the relationship between the methylation status of prostanoid receptor genes and recurrence in HNSCC. These results provide new perspectives for the development of molecular targeted treatment approaches.

Keywords: Prostanoid receptor genes, GPCRs, TET, Epigenetic markers, Q-MSP

\section{Background}

Clinical and epidemiological evidence suggests that chronic inflammation is a major risk factor for head and neck malignancies [1]. For example, patients with

\section{${ }^{*}$ Correspondence: kiyoshim@hama-med.ac.jp}

Department of Otolaryngology/Head and Neck Surgery, Hamamatsu University School of Medicine, 1-20-1 Handayama, Shizuoka 431-3192, Japan persistent human papilloma virus (HPV) infection, Epstein-Barr virus infection, or chronic inflammation (as observed in individuals with a cigarette smoking habit) face an increased lifetime risk for oropharyngeal cancer, nasopharyngeal cancer, or laryngeal cancer, respectively [2, 3]. Many studies have focused on cytokines and chemokines as mediators connecting chronic

(c) The Author(s) 2020. This article is licensed under a Creative Commons Attribution 4.0 International License, which permits use, sharing, adaptation, distribution and reproduction in any medium or format, as long as you give appropriate credit to the original author(s) and the source, provide a link to the Creative Commons licence, and indicate if changes were made. The images or other third party material in this article are included in the article's Creative Commons licence, unless indicated otherwise in a credit line to the material. If material is not included in the article's Creative Commons licence and your intended use is not permitted by statutory regulation or exceeds the permitted use, you will need to obtain permission directly from the copyright holder. To view a copy of this licence, visit http://creativeco mmons.org/licenses/by/4.0/. The Creative Commons Public Domain Dedication waiver (http://creativecommons.org/publicdomain/ zero/1.0/) applies to the data made available in this article, unless otherwise stated in a credit line to the data. 
inflammation to head and neck cancer, but little is known about the involvement of prostanoid receptors [4]. To improve the survival rate for head and neck cancer, precision medicine approaches, improvements in diagnosis and prognosis, as well as the identification of novel targets and treatment strategies with minimal side effects are required.

G protein-coupled receptors (GPCRs) are the largest class of cell-surface receptors and are involved in many cancers, including head and neck squamous cell carcinoma (HNSCC) $[5,6]$. GPCRs are modulated by a variety of endogenous and synthetic ligands and are major drug targets [7]. Key therapeutic applications involving GPCRs include opioid analgesics, antihistamines, anticholinergics, typical and atypical antipsychotics, antimigraine drugs, $\beta 2$-agonists for asthma, and anti-hypertensives [8]. However, anti-cancer drugs that specifically target GPCRs are not currently available [9]. The prostanoid receptors represent the most notable family of validated pharmacological targets in a variety of diseases, including cancer [10].

Prostanoids derived from arachidonic acid through the cyclooxygenase (COX) pathway are particularly relevant. Prostaglandin H2 (PGH2) is the common cyclic-peroxide intermediate in the biosynthesis of prostanoids derived from arachidonic acid [11]. Prostanoids are a group of lipid mediators that include prostaglandins (PG) and thromboxanes (TX) [12]. Fatty acid COX converts arachidonic acid to PGH2, from which further prostanoids, PGD2, PGE2, PGF2 $\alpha$, PGI2 (prostacyclin), and thromboxane A2 (TXA2), may be enzymatically derived [13]. All nine prostanoid receptor genes [prostaglandin D2 receptors (PTGDR1 and PTGDR2), four prostaglandin E2 receptors (PTGER1, PTGER2, PTGER3 and PTGER4), the prostaglandin $\mathrm{F}$ receptor (PTGFR), the prostaglandin 12 receptor $(P T G I R)$, and thromboxane A2 receptor $(T B X A 2 R)]$ encode neuropeptide receptors and belong to the GPCR Class A $\alpha$ subgroup [14]. These nine prostanoid receptor genes have been implicated in the development of multiple types of cancer, but studies of the methylation status of all nine genes and their roles in the prognosis of HNSCC are lacking.

In this study, we provide that associations between the methylation status of nine prostanoid receptor genes and clinicopathological characteristics (e.g., tumour location and recurrence events) were also assessed. To our knowledge, this study is the first to link prostanoid receptor gene methylation to the genesis of HNSCC.

\section{Methods}

\section{Tumour samples}

Surgical HNSCC tumour and matched adjacent nontumour tissues were obtained from 274 patients who underwent surgical resection at the Department of Otolaryngology/Head and Neck Surgery, Hamamatsu University School of Medicine (Hamamatsu, Shizuoka, Japan). Written informed consent was obtained from individual patients before surgery and the experimental protocol was approved by the Hamamatsu University School of Medicine (date of board approval: October 2, 2015, ethics code: 25-149). The ratio of males to females was $227: 47$. The mean age was 65.2 years (range, 32 to 90 years). Primary head and neck tumours included 69 hypopharyngeal carcinomas, 51 laryngeal carcinomas, 79 oropharyngeal carcinomas, and 75 oral cavity carcinomas (Additional file 1: Table S1).

\section{DNA extraction and modification}

DNA extraction from fresh tissue was performed using a QIAamp DNA Mini Kit (Qiagen, Hilden, Germany). Sodium bisulphite conversion was performed using the MethylEasy Xceed Rapid DNA Bisulfite Modification Kit (TaKaRa, Tokyo, Japan) following the manufacturer's protocol.

\section{Quantitative methylation-specific PCR analysis (Q-MSP)}

Aberrant DNA methylation, which often occurs around the transcription start site (TSS) within a CpG island, was evaluated by Q-MSP. The sequences of primers used in this study are shown in Additional file 2: Table S2. Exon one or two and CpG sites within view of the promoter region relative to the TSS are presented in Additional file 3: Figure S1. A standard curve for Q-MSP was constructed by plotting five serially diluted standard solutions of EpiScope Methylated HeLa gDNA (TaKaRa). The normalized methylation value (NMV) was defined as follows: $\mathrm{NMV}=$ (prostanoid receptor gene-S/prostanoid receptor gene-FM)/(ACTB-S/ACTB-FM), where prostanoid receptor gene-S and prostanoid receptor gene-FM represent target gene methylation levels in the tumour sample and in the universal methylated DNA control, respectively. ACTB-S and ACTB-FM correspond to $\beta$-actin (ACTB) in the sample and the universally methylated DNA, respectively [15].

\section{Detection of high-risk HPV DNA by PCR}

To identify the HPV types, samples were also subjected to PCR using specific primers for HPV types 16, 18, 31, $33,35,52$, and 58 . The prevalence of HPV DNA was examined using the PCR HPV Typing Set (TaKaRa).

\section{ELISA for 5-hmC quantification}

The $5 \mathrm{hmC}$ content of genomic DNA was determined with a Quest 5-hmC DNA ELISA Kit (Zymo Research, Irvine, CA, USA), according to the manufacturer's instructions. The amount of $5-\mathrm{hmC}$ was calculated as a 
percentage based on a standard curve generated using kit controls.

\section{RNA extraction and quantitative reverse transcription PCR (qRT-PCR)}

Total RNA was isolated using an RNeasy Plus Mini Kit (Qiagen, Hilden, Germany); cDNA was synthesized using a ReverTra Ace qPCR RT Kit (Toyobo, Tokyo, Japan). DNMT3A, DNMT3B, and GAPDH mRNA expression levels were measured via qRT-PCR using SYBR Premix Ex Taq (Takara Bio Inc., Tokyo, Japan), the Takara Thermal Cycler Dice Real Time System TP8000 (Takara Bio Inc.), and the primer sets presented in previously reports [16].

\section{Data mining in the Cancer Genome Atlas (TCGA)}

MethHC (http://methhc.mbc.nctu.edu.tw/php/index .php) was used to extract data from TCGA (available in August 2019). DNA methylation of prostanoid receptor genes was measured using the Illumina Infinium Human Methylation $450 \mathrm{~K}$ BeadChip. The methylation score for each $\mathrm{CpG}$ site was estimated as the $\beta$-value, which ranges from 0 to 1 , corresponding to unmethylated and completely methylated DNA, respectively. In addition, RNAseq data of PTGDR1, PTGER1, PTGER2, PTGER3, PTGER4, PTGFR, PTGIR, TBXA2R, IL-6, IL-11 and $R A N K$ were obtained from the TCGA data portal (https ://tcga-data.nci.nih.gov/tcga/).

\section{Data analysis and statistics}

A receiver operator characteristic (ROC) curve analysis of target genes was performed using the NMVs for 36 matched paired HNSCC and normal mucosal samples and the Stata/SE 13.0 system (Stata Corporation, College Station, TX, USA). To determine the area under the ROC curve, the true positive rate (Sensitivity) was plotted as a function of the false positive rate $(1-$ Specificity) for different cut-off points, and the NMV thresholds were calculated for each target gene. Cut-off values showing the greatest accuracy were determined based on sensitivity/specificity, as indicated in Additional file 4: Table S3. The MI was defined as the number of genes with promoter methylation [17]. Student's $t$-tests were performed to evaluate the associations between clinical variables and MI. Disease-free survival (DFS) was investigated using the Kaplan-Meier method and the log-rank test. The probability of survival can be evaluated by generating a Kaplan-Meier curve. A Cox's proportional hazards regression analysis that included age ( $\geq 65$ vs. $<65$ years), sex, alcohol intake, smoking status, and tumour stage (III vs. III-IV) and methylation status was used to identify the multivariate predictive value of prognostic factors. A value of $\mathrm{P}<0.05$ was considered statistically significant.

\section{Results}

Characterisation of 36 matched paired head and neck tumour samples and adjacent noncancerous mucosal samples

Promoter hypermethylation of nine prostanoid receptor genes exhibited distinct ROC curve profiles, which clearly differentiate cancer tissues from normal tissues (Additional file 5: Fig. S2). A specimen was classified as methylated when its NMV exceeded 0.161, 0.123, 0.048, 0.161, 0.502, 0.419, 0.368, 0.109, and 0.082 for PTGDR1, PTGDR2, PTGER1, PTGER2, PTGER3, PTGER4, PTGFR, $P T G I R$, and TBXA2R, respectively (Additional file 4: Table S3). Methylation levels of all prostanoid receptor genes in primary HNSCCs were significantly higher than those in matched paired normal mucosal tissues, except for PTGER3 (Additional file 6: Fig. S3).

\section{Analysis of methylation status in HNSCC tissue samples}

A Q-MSP analysis of the methylation status of nine prostanoid receptor genes was performed using 274 primary HNSCC samples. The methylation frequencies were as follows: PTGDR1 (43.8\%), PTGDR2 (18.2\%), PTGER1 (25.5\%), PTGER2 (17.5\%), PTGER3 (41.2\%), PTGER4 (8.0\%), PTGFR (19.3\%), PTGIR (20.4\%), and TBXA2R (11.3\%) (Fig. 1a). The average MI per sample was $2.05 \pm 2.59$ (range 0 to 9) (Fig. 1b). No significant differences in MI were observed with respect to the age at disease onset, sex, alcohol consumption, smoking status, tumour size, lymph node status, clinical stage, or HPV status (Fig. 1c). We analysed the relationships of the methylation status of each prostanoid receptor gene with the clinical features of patients with HNSCC. PTGFR methylation was significantly correlated with age at onset $(\mathrm{P}=0.043)$. Methylation levels of PTGDR2, PTGER1, and PTGFR promoters were associated with alcohol exposure $(\mathrm{P}=0.042, \mathrm{P}=0.04$, and $\mathrm{P}=0.049$, respectively). There was an association between methylation of the PTGDR1 and PTGER3 promoters and HPV status $(\mathrm{P}=0.004$ and $\mathrm{P}=0.005$, respectively). We found that the promoter methylation of all prostanoid receptor genes with the exception of $T B X A 2 R$ was associated with recurrence events (Table 1 ).

\section{Comparison of methylation frequencies between nine prostanoid receptor genes and ten-eleven translocation (TET) family genes}

Mean differences in the MI of nine prostanoid receptor genes based on TET gene methylation events are illustrated in Fig. 2a. The MI was significantly higher in patients with methylation events at all TET genes $(4.84 \pm 2.73)$, two TET gene methylation events $(2.92 \pm 3.09)$, and one TET gene methylation event $(1.94 \pm 2.03)$ than in patients with no TET gene methylation events $(0.39 \pm 1.05 ; \mathrm{P}<0.01$ for all comparisons) (Fig. 2b). 


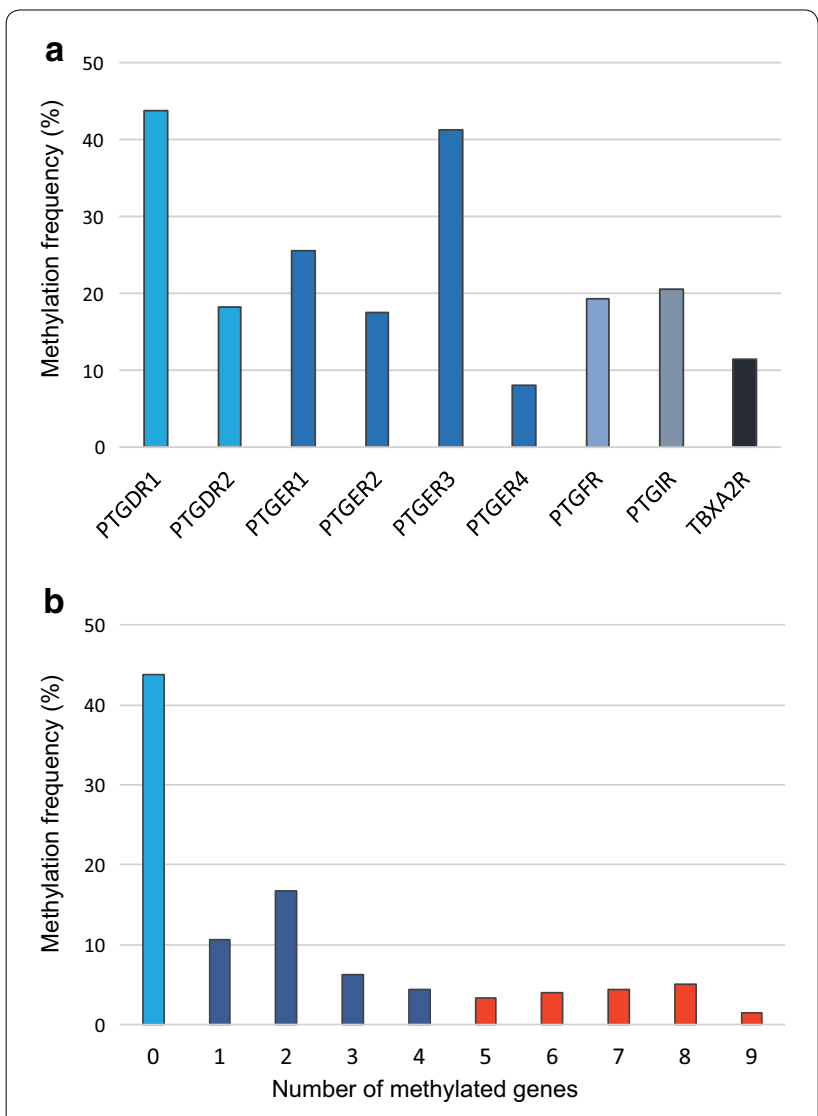

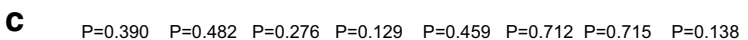

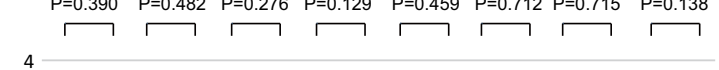

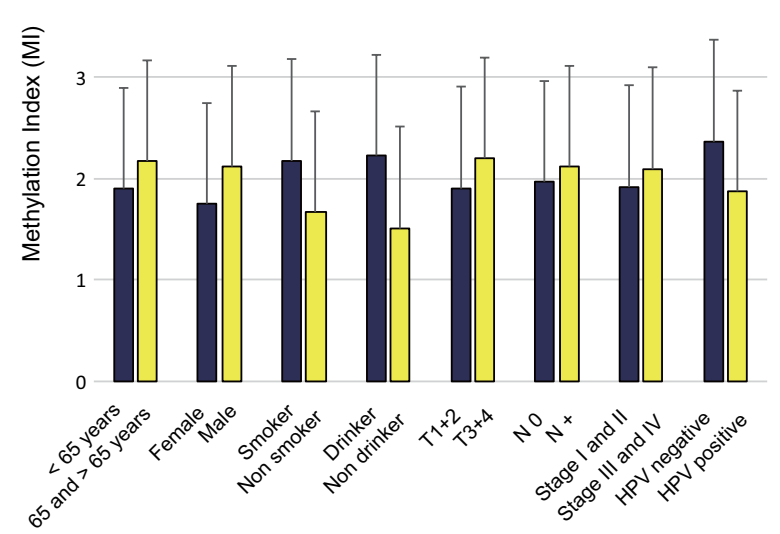

Fig. 1 Methylation of nine prostanoid receptor gene promoters in 274 HNSCC samples. a Bar graph showing methylation frequencies of the nine genes. $\mathbf{b}$ Bar graph showing the percentage of tumours with zero to nine methylated target genes. c Bar graph showing the MI according to selected clinical parameters. Differences in mean MI for each parameter were determined by Student's t-tests

\section{Survival analysis}

The results of a Kaplan-Meier analysis of DFS are shown in Fig. 3. DFS did not differ between patients with methylated and unmethylated genes (Fig. 3d-h), with several notable exceptions, i.e., DFS was significantly shorter when the PTGDR1 (log-rank test, $\mathrm{P}=0.019$ ), PTGDR2 (log-rank test, $\mathrm{P}=0.025)$, PTGER1 (log-rank test, $\mathrm{P}=0.024)$, and $T B X A 2 R(\log$-rank test, $\mathrm{P}=0.041)$ promoters were methylated (Fig. $3 \mathrm{a}-\mathrm{c}, \mathrm{i}$ ). DFS in patients with 5-9 methylated genes was lower than that in the group with $0-4$ methylated genes (35.4\% versus 59.0\%; log-rank test, $\mathrm{P}=0.007$; Fig. 3 j, Additional file 7 : Table S4).

\section{Site-specific analysis of the methylation status}

Site-specific methylation frequencies across nine genes for the hypopharynx, larynx, oropharynx, and oral cavity are shown in Fig. 4a. MI levels were significantly higher in patients with hypopharyngeal cancer than in patients with oral cavity cancer $(P=0.020)$ (Fig. $4 b)$. Among 69 cases with hypopharyngeal cancer, the DFS rate in those with PTGDR1 methylation was similar to that in the unmethylated group (log-rank test, $\mathrm{P}=0.011$; Additional file 8: Fig. S4A). Patients with laryngeal cancer and methylated PTGER4 promoters had a relatively short DFS (log-rank test, $\mathrm{P}=0.020$; Additional file 8: Fig. S4B). Additional analysis including only patients with oropharyngeal cancer $(\mathrm{n}=79)$ revealed a shorter DFS for methylated than for unmethylated PTGIR and TBXA2R (log-rank test, $\mathrm{P}=0.003$ and $\mathrm{P}=0.009$, respectively; Additional file 8: Fig. S4C, D).

\section{Stratification analysis}

The relation between the methylation status and risk of recurrence was analysed by a multivariate analysis using a Cox proportional hazards regression model adjusted for age, sex, smoking status, alcohol consumption, and clinical stage. For 274 patients with PTGDR1 promoter methylation, the adjusted odds ratio (OR) for recurrence was 1.58 [95\% confidence interval (CI) $1.06-2.33, \mathrm{P}=0.023]$. In patients with hypopharyngeal cancer, PTGDR1 promoter methylation was significantly associated with recurrence $(\mathrm{OR}=2.76,95 \%$ CI $1.23-6.18, \mathrm{P}=0.014)$. For patients with laryngeal cancer with a methylated PTGER4 promoter, the OR was 5.04 (95\% CI 1.03-24.70; $\mathrm{P}=0.046)$. Methylation statuses of the PTGIR and TBXA2R promoters were positively correlated with recurrence in patients with oropharyngeal cancer (OR, 2.99; 95\% CI 1.13-7.92; $\mathrm{P}=0.028$ and $\mathrm{OR}, 5.21 ; 95 \%$ CI $1.63-16.67 ; \mathrm{P}=0.006$, respectively) (Fig. 5).

\section{Comparison of methylation frequencies between nine prostanoid receptor genes and other epigenetic factors}

The 5-hmC level showed the greatest decrease when prostanoid receptor genes were 9 to 5 methylation events 


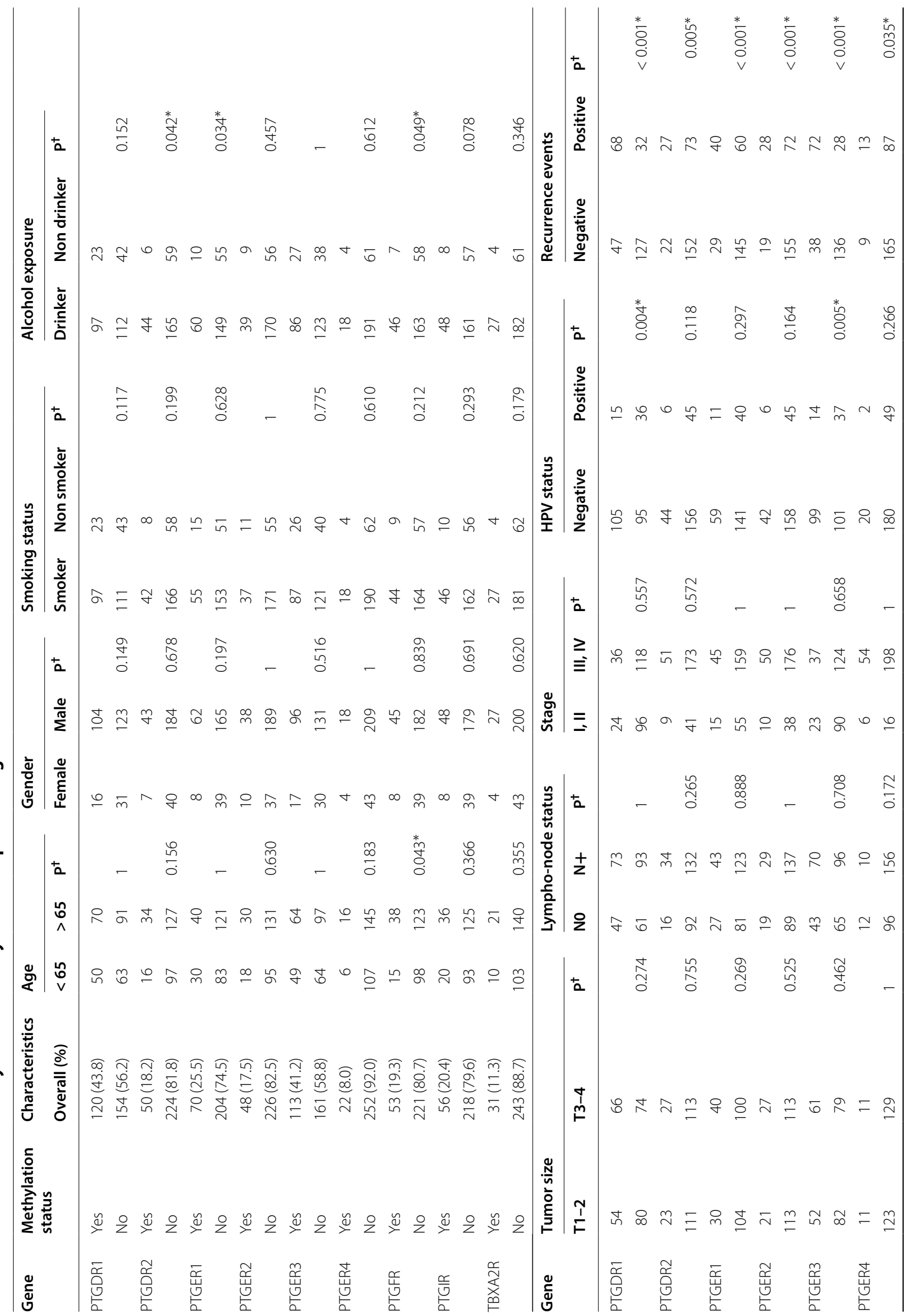




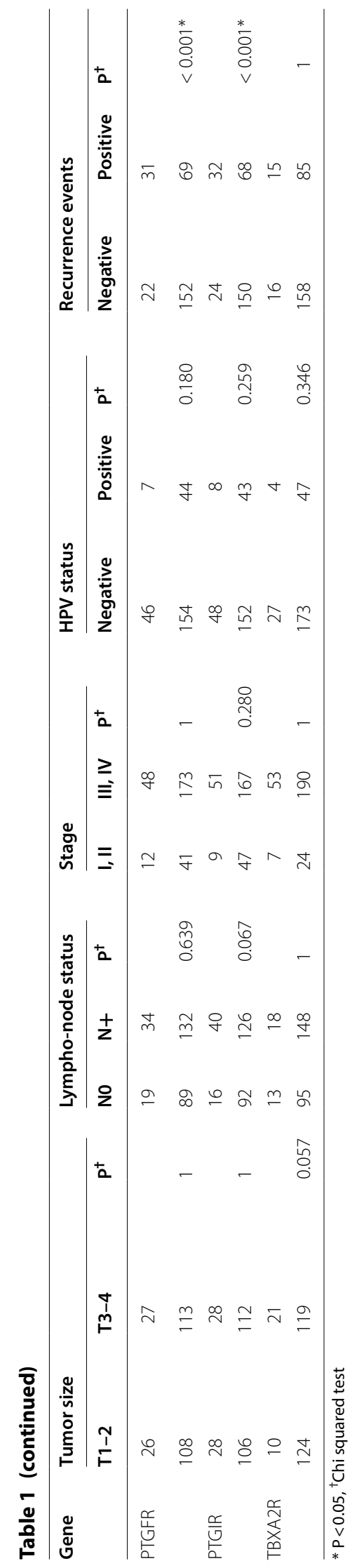




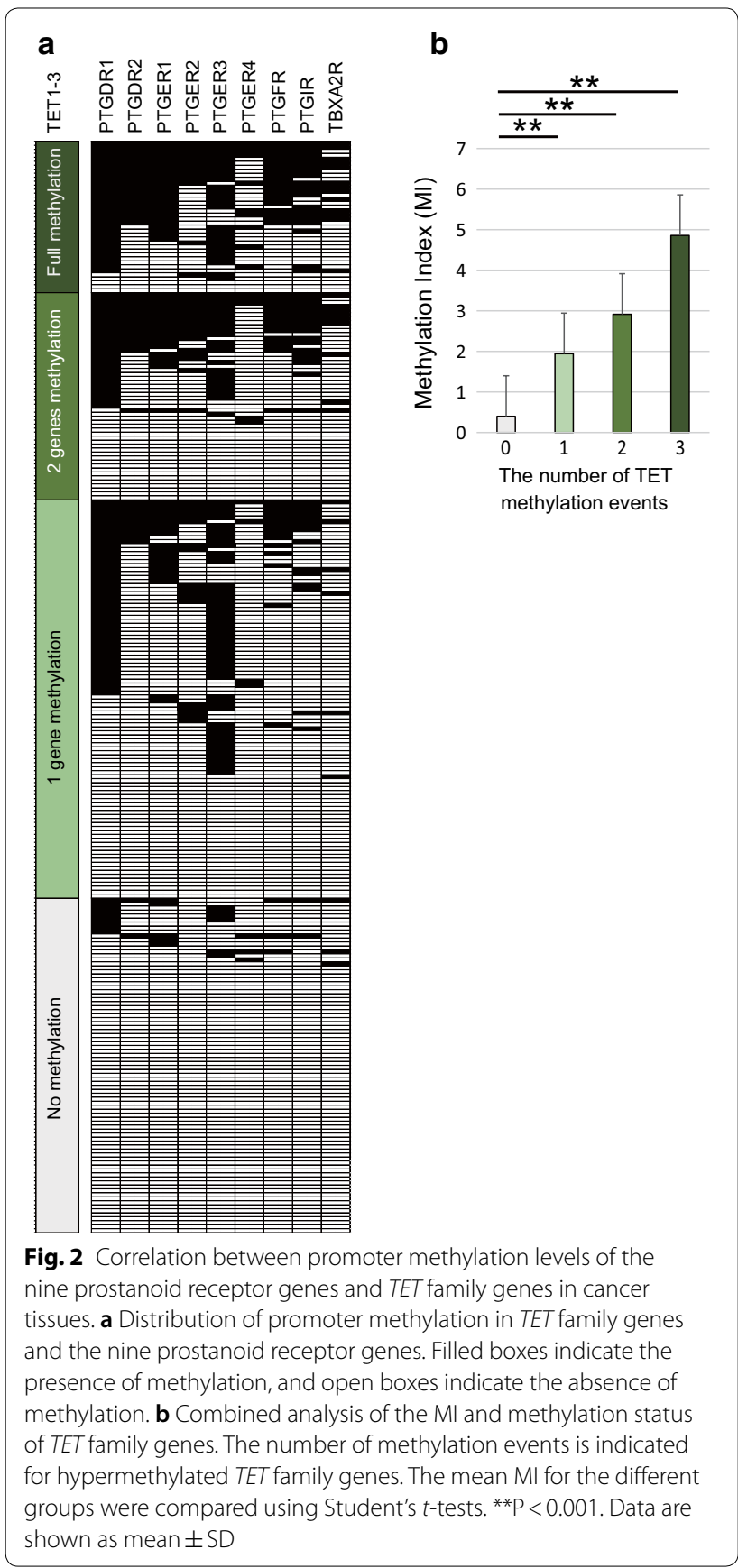

$(\mathrm{P}=0.035$; Additional file 9: Fig. S5A). The DNMT3A mRNA expression levels were significantly higher in groups with 9 to 5 methylation and 4 to 1 methylation $(\mathrm{P}=0.026$ and $\mathrm{P}=0.029$, respectively; Additional file 9: Fig. S5B). The DNMT3B mRNA expression was significantly correlated with both 9 to 5 methylation and 4 to 1 methylation $(\mathrm{P}=0.013$ and $\mathrm{P}=0.020$, respectively; Additional file 9: Fig. S5C).
Analysis of methylation and expression data from TCGA The methylation status of prostanoid receptor gene promoters was estimated in an additional 516 HNSCC samples and 50 normal samples from TCGA. The average $\beta$-values (indicating promoter methylation) for the nine genes were significantly higher in the HNSCC samples than in the normal samples $(\mathrm{P}<0.05)$, except for PTGER4 and TBXA2R (Additional file 10: Fig. S6). The expression of prostanoid receptor genes were significantly higher in $I L-6$ mRNA high expression group $(\mathrm{P}<0.05)$, except for PTGER1 gene. $I L-11$ expression was positively correlated with PTGDR1 and TBXA2R expression $(\mathrm{P}=0.001$ and $\mathrm{P}<0.001$, respectively). Expression of $R A N K L$ was concurrently associated with all prostanoid receptor genes expression (Additional file 11: Table S5).

\section{Discussion}

Epigenetic modifications of prostanoid receptor genes may contribute to tumour development and recurrence. We analysed the methylation statuses of genes encoding neuropeptide GPCRs in 274 HNSCCs originating in the hypopharynx, larynx, oropharynx, or oral cavity. We also compared the methylation status of genes in matched HNSCC and normal samples using data from TCGA. We found that the aberrant methylation of some prostanoid receptor gene promoters is positively correlated with recurrence in patients with HNSCC. In addition, a site-specific analysis revealed that abnormal CpG island hypermethylation was independently associated with aggressive clinical behaviour.

Cancer may be related to chronic inflammation associated with persistent infections, immune-mediated damage, or prolonged exposure to irritants. Genetic and epigenetic alterations underlying carcinogenesis inevitably modify tissue homeostasis and may induce a chronic inflammatory response. Over 20 years ago, non-steroidal anti-inflammatory drugs (NSAIDs) were reported to have anti-colon cancer effects [18]. NSAIDs, which are potent inhibitors of COX, exert chemopreventive effects in cancer development [19]. Numerous epidemiological studies have shown that the regular intake of the NSAID aspirin, an inhibitor of COXs, substantially reduces both the incidence and progression of several prevalent cancers [20]. Abundant epidemiological and preclinical/clinical studies have demonstrated that celecoxib, a specific COX-2 inhibitor, is related to the suppression of cancer cell proliferation and a decrease in cancer incidence [21]. COX-1 is constitutively expressed in many tissues and regulates basal levels of prostaglandins [22]. COX-2 is responsible for the release of prostaglandins after an infection, injury, or in cancer development [23]. In HNSCC, IL-1 released by tumour cells plays a key role in inducing 

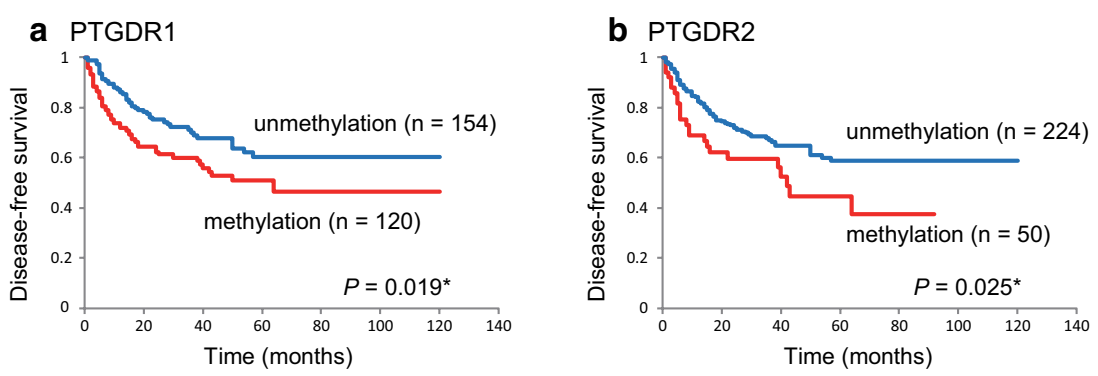

\section{c PTGER1}

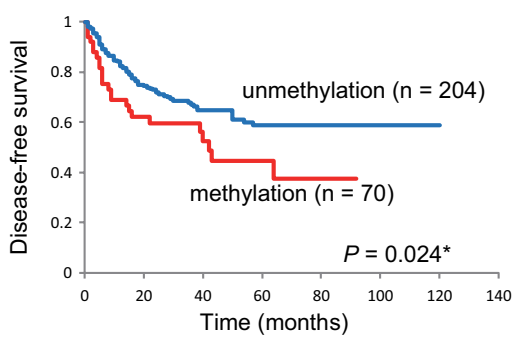

d PTGER2

\section{e PTGER3}
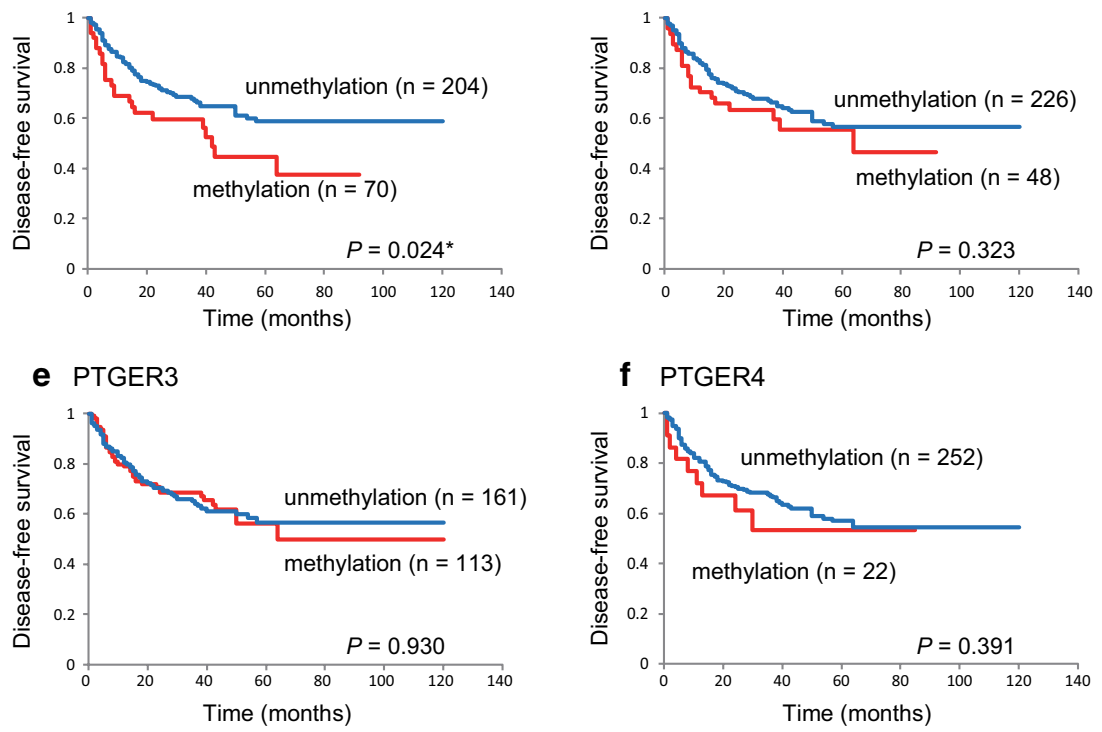

\section{f PTGER4}

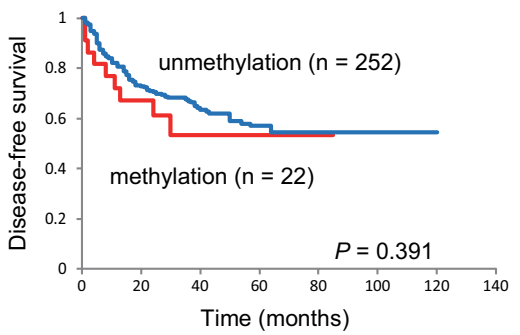

\section{g PTGFR}

\section{h PTGIR}
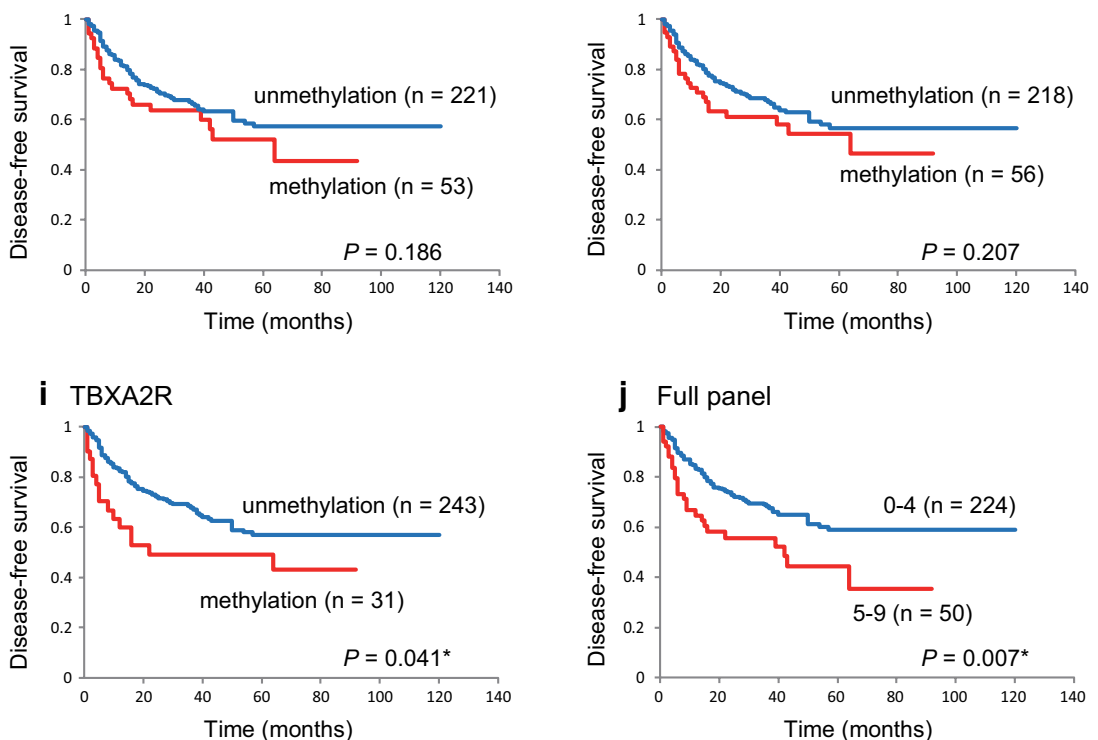

Fig. 3 Kaplan-Meier survival curves based on 274 patients with HNSCC according to the methylation status of the nine prostanoid receptor genes. DFS with respect to a PTGDR1, b PTGDR2, $\mathbf{c}$ PTGER1, d PTGER2, e PTGER3, f PTGER4, $\mathbf{g}$ PTGFR, $\mathbf{h}$ PTGIR, and $\mathbf{i} T B X A 2 R$ in the case of methylated (red lines) and unmethylated (blue lines) genes. $\mathbf{j}$ Combined analysis of the nine genes. Blue line: patients with 0-4 methylated genes; red line: patients with 5-9 methylated genes. ${ }^{*} \mathrm{P}<0.05$ 
a

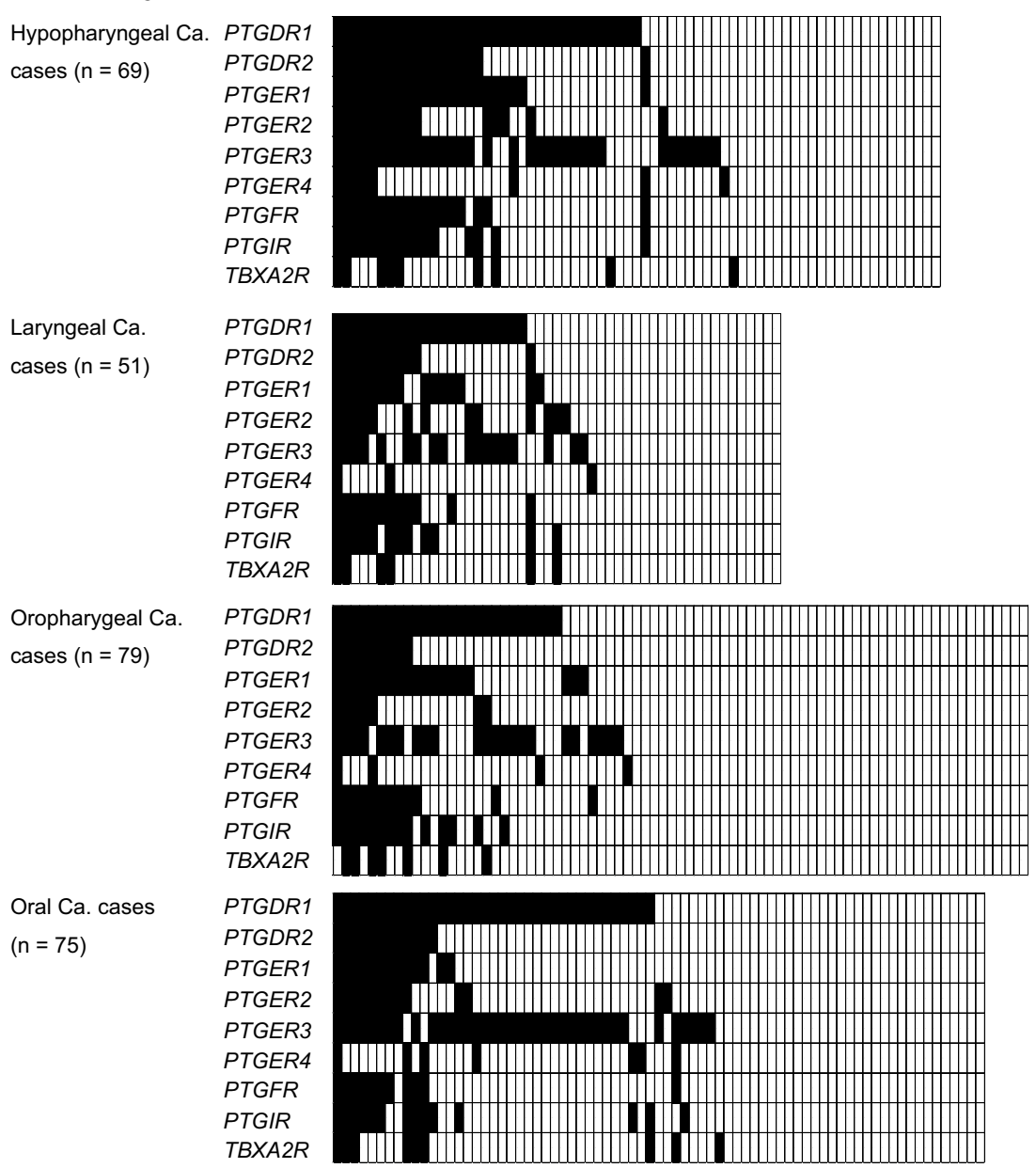

b

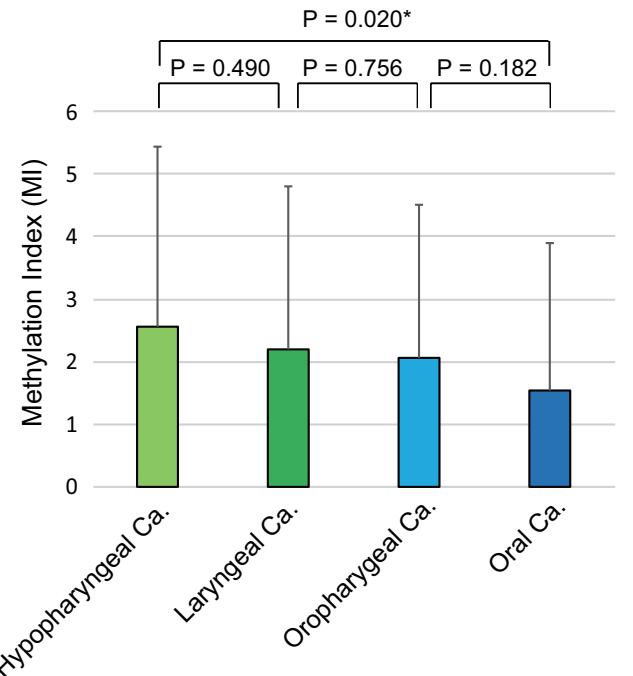

Fig. 4 Site-specific methylation frequencies for nine prostanoid receptor genes. a Comparison of methylation statuses of the promoters of the nine prostanoid receptor genes in patients with hypopharyngeal, laryngeal, oropharyngeal, or oral cancer. Filled boxes indicate the presence of methylation, and open boxes indicate the absence of methylation. b The mean MI values for various groups were compared using Student's $t$-tests. ${ }^{*} \mathrm{P}<0.05$ 


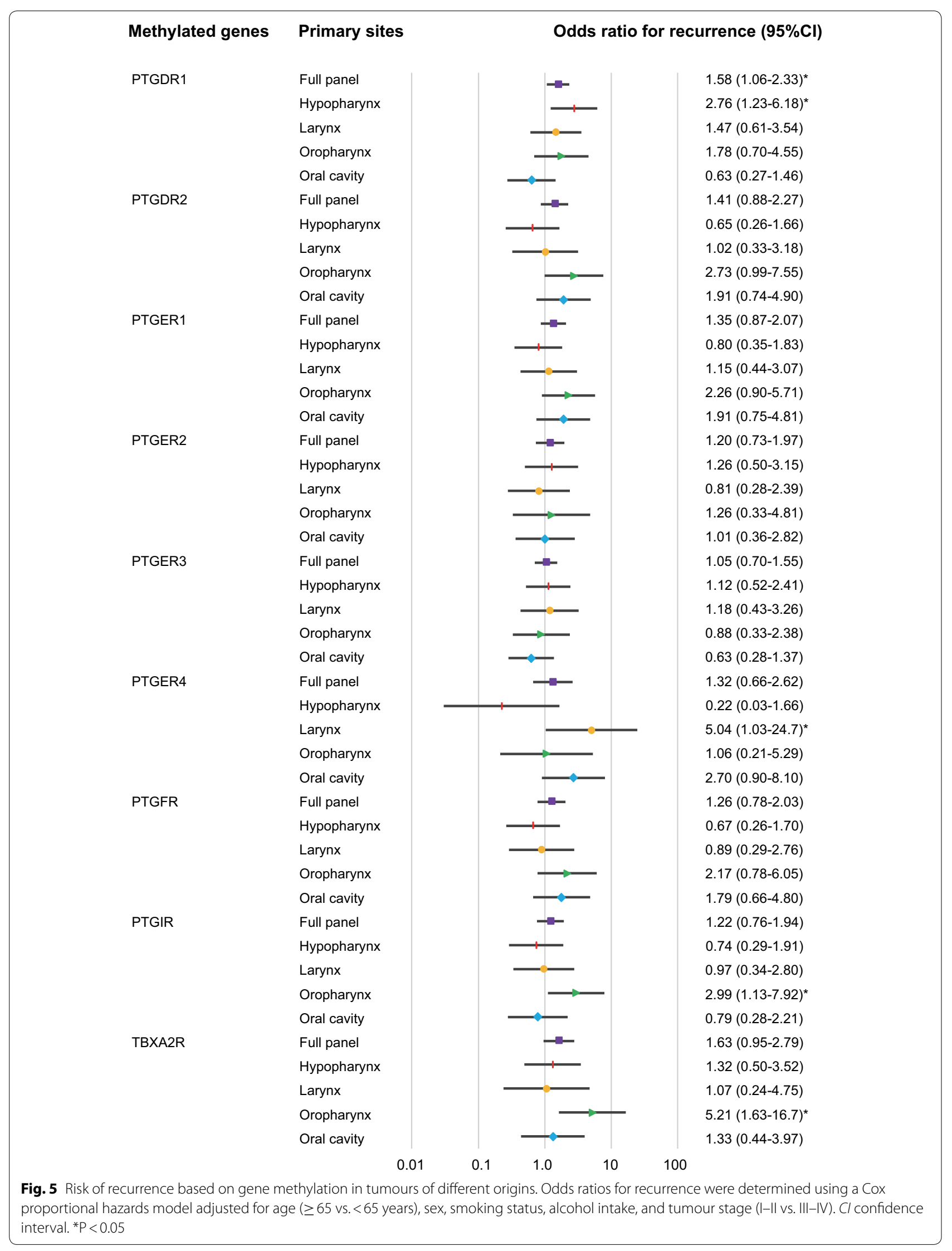


the expression of COX-2 in fibroblasts [11]. Secretion of TGF- $\beta$ and PGE2 by the HNSCC cells was increased following EGFR inhibition [24]. IL-6, TNF-a and PGE2 produced by primary oral keratinocytes and carcinoma cells may induce oral mucosal inflammation [25]. Recent studies continue to support the prostanoid pathway as a promising target for future HNSCC therapies.

Prostanoids, including PGD2, PGE2, PGF2 $\alpha$, PGI2, and TXA2, activate nine GPCRs, namely PTGDR1, PTGDR2, PTGER1, PTGER2, PTGER3, PTGER4, PTGFR, PTGIR, and TBXA2R. PTGDR1 downregulation by DNA hypermethylation is correlated with colorectal cancer development [26, 27]. PTGDR1 methylation from cervical scraping is a promising marker of endometrial cancer and ovarian cancer [28]. PTGER1 shows a strong association with DNA methylation in non-functioning adrenocortical adenoma [29]. The expression of PTGER2 is often silenced in neuroblastoma cell lines by epigenetic mechanisms [30]. Increased DNA methylation of PTGER2 is associated with the progression of neuroblastomas [30], non-small cell lung cancer [31], and cervical cancer tissue [32], suggesting that the aberrant methylation of this gene regulates cell proliferation. Cebola et al. detected PTGER3 and PTGFR hypermethylation in a high proportion of colorectal cancer cases, suggesting that DNA methylation is an important mechanism involved in the deregulation of this pathway [33]. The measurement of PTGER4 methylation in plasma DNA obtained by minimally invasive sampling can be used to detect malignant lung disease [34]. The loss of methylation and activation of PTGER4 can explain the acquisition of endocrine therapy resistance and is a therapeutic target for breast cancer [35].

Cancers of the upper aerodigestive tract account for the majority of squamous cell carcinomas, which develop in the epithelial linings of the oral cavity, pharynx, and larynx [36]. There are several subclassifications based on anatomic location, aetiology, and molecular findings [37]. Head and neck cancers arise from a multistep process involving the accumulation of genetic and epigenetic alterations [38]. DNA methylation is a frequent and key epigenetic mechanism underlying the regulation of processes associated with neoplastic transformation [38]. Recently, TET proteins have been identified as important epigenetic modifiers via their dioxygenase activity [39]. TET expression and activity are inhibited by genetic mutations and high methylation of their own promoters [40]. Our data showed that increased DNA methylation of TET genes is correlated with the accumulation of prostanoid receptor genes with aberrant methylation; this may be a meaningful DNA methylation event in HNSCC progression. Furthermore, the groups of high MI were extremely low of $5 \mathrm{hmC}$ levels and was correlated with increasing expression of DNMT3A and DNMT3B. To our knowledge, our study is the first to suggest that PTGDR1, PTGER4, PTGIR, and TBXA2R methylation is associated with worse DFS and may be a critical event in hypopharyngeal cancers, laryngeal cancers, oropharyngeal cancers, and oral cancers, respectively. However, our results obtained from human specimens and highthroughput profiling platforms may be susceptible to measurement bias from various sources. The current study continues to support the prostanoid receptor as a promising target for future HNSCC therapies.

We systematically evaluated the methylation status of the promoters of nine prostanoid receptor genes and the relationship between methylation and clinical characteristics in HNSCC samples. We identified a novel prognostic biomarker based on promoter DNA methylation changes in operable HNSCC to identify patients at high risk of recurrence and provide complementary epigenetic characterization of this tumour type. Collectively, these data demonstrate the functional roles of the epigenetic regulation of prostanoid receptors and show that these loci are potential targets for epigenetic therapies for inflammatory disorders, such as HNSCC.

\section{Conclusion}

We determined the relationship between the methylation status of prostanoid receptor genes and recurrence in HNSCC, providing new perspectives for the development of molecular targeted therapeutic approaches. To our knowledge, our study provides the first evidence for an association between PTGDR1, PTGER4, PTGIR, and $T B X A 2 R$ methylation and worse survival in hypopharyngeal cancers, laryngeal cancers, oropharyngeal cancers, and oral cancers, respectively. This study involving human specimens and high-throughput profiling platforms may be susceptible to measurement bias from various sources; accordingly the use of methylation markers in clinical practice requires further testing in prospective studies with larger HNSCC cohorts.

\section{Supplementary information}

Supplementary information accompanies this paper at https://doi. org/10.1186/s12967-020-02214-1.

Additional file 1: Table S1. Baseline characteristics of the HNSCC patients.

Additional file 2: Table S2. Q-MSP primer list.

Additional file 3: Fig. S1. Schematic representation of PTGDR1, PTGDR2, PTGER1, PTGER2, PTGER3, PTGER4, PTGFR, PTGIR and TBXA2R genes. CpG sites are within the expanded views of the promoter region. Vertical lines, individual CPG sites; black box, relative location of the primers used for Q-MSP; bent arrow, translation start site (ATG).

Additional file 4: Table S3. Results of the ROC curve analysis, the sensitivity, specificity, and cutoff value. 
Additional file 5: Fig. S2. Receiver operating characteristic (ROC) curves for the methylation markers in cancer tissue versus adjacent normal mucosal tissue. Based on the ROC curve analysis, Area Under Curves (AUCS) are 0.6767 for PTGDR1 (A), 0.6265 for PTGDR2 (B), 0.6574 for PTGER1 (C), 0.6154 for PTGER2 (D), 0.4784 for PTGER3 (E), 0.5405 for PTGER4 (F), 0.6289 for PTGFR (G), 0.6736 for PTGIR (H) and 0.6605 for TBXA2R (I).

Additional file 6: Fig. S3. Hypermethylation patterns in 36 matched pairs of head and neck tumors and adjacent normal mucosal tissues. The NMVs for the PTGDR1 (A), PTGDR2 (B), PTGER1 (C), PTGER2 (D), PTGER3 (E), PTGER4 (F), PTGFR (G), PTGIR (H) and TBXA2R (I) promoters were significantly higher in head and neck tumor tissues ( $T$ ) than in paired adjacent normal mucosal tissue (N). The differences were significant as determined by the Student's t-test. ${ }^{*} P<0.05$

Additional file 7: Table S4. Results of log-rank tests for effect of number of methylated genes on disease free survival in 274 HNSCC.

Additional file 8: Fig. S4. Kaplan-Meier survival curves. Kaplan-Meier survival curves for PTGDR1 in (A) patients with hypopharyngeal cancer $(n=69)$, for PTGER4 in (B) patients with laryngeal cancer $(n=51)$, and for PTGIR and TBXA2R in ( $C$ and $D)$ patients with oropharyngeal cancer $(n=79)$. The log-rank test was used to compare the survival times between patients with methylated (red lines) and unmethylated (blue lines) genes. ${ }^{*} P<0.05$.

Additional file 9: Fig. S5. Comparison of methylation frequencies between nine prostanoid receptor genes and other epigenetic factors. (A) $5 \mathrm{hmC}$ levels, (B) DNMT3A mRNA levels, (C) DNMT3B mRNA levels. ${ }^{*} \mathrm{P}<0.05$. The data are shown as the mean $\pm \mathrm{SE}$.

Additional file 10: Fig. S6. Methylation status of the five neuropeptide receptor genes in HNSCC and normal samples in TCGA database. The methylation data for PTGDR1, PTGDR2, PTGER1, PTGER2, PTGER3, PTGER4, PTGFR, PTGIR and TBXA2R in HNSCC and normal samples were collected from TCGA database. * $\mathrm{P}<0.05$

Additional file 11: Table S5. Distribution of expression levels in TCGA cohort of HNSCC.

\section{Abbreviations}

COX: cyclooxygenase; DFS: disease-free survival; GPCRs: G protein-coupled receptors; HNSCC: head and neck squamous cell carcinoma; HPV: human papilloma virus; NMV: normalized methylation value; NSAIDs: non-steroidal antiinflammatory drugs; PG: prostaglandins; PTGDR: prostaglandin D2 receptors; PTGER: prostaglandin E2 receptors; PTGFR: prostaglandin F receptor; PTGIR: prostaglandin 12 receptor; Q-MSP: Quantitative methylation-specific PCR; ROC: receiver-operator characteristic;TCGA: Cancer Genome Atlas; TBXA2R: thromboxane A2 receptor; TET: ten-eleven translocation; TSS: transcription start site; TX: thromboxanes; TXA2: thromboxane A2.

\section{Acknowledgements}

We would also like to thank the research, laboratory and clinical staff who supported this study.

\section{Authors' contributions}

KM and YM conceived the study. KM and YM designed the experiments. MM, $\mathrm{SY}, \mathrm{Al}, \mathrm{DM}, \mathrm{RI}, \mathrm{JK}, \mathrm{YY}, \mathrm{SE}$ and $\mathrm{HM}$ analyzed the data and prepared figures and tables. All authors wrote the manuscript, reviewed its drafts, and agreed with its submission. All authors read and approved the final manuscript.

\section{Funding}

This study was funded by a Grant-in-Aid for Scientific Research (Nos. 16K11228, 16K20239, 17K16904, 17K16903, 19K09866, 19 K09906 and 19K18728) from the Ministry of Education, Culture, Sports, Science, and Technology of Japan.

\section{Availability of data and materials}

The datasets during and/or analyzed during the current study available from the corresponding author on reasonable request.

\section{Ethics approval and consent to participate}

The research methodology employed in this study was approved by The Institutional Review Board of the Hamamatsu University School of Medicine All study subjects provided written informed consent.

\section{Consent for publication}

Consent for publication was obtained from all patients.

\section{Competing interests}

The authors declare that they have no competing interests.

Received: 8 November 2019 Accepted: 4 January 2020

Published online: 21 January 2020

\section{References}

1. Tezal M. Interaction between chronic inflammation and oral HPV infection in the etiology of head and neck cancers. Int J Otolaryngol. 2012;2012:575242.

2. Guidry JT, Scott RS. The interaction between human papillomavirus and other viruses. Virus Res. 2017;231:139-47.

3. Stell PM. Smoking and laryngeal cancer. Lancet. 1972;1(7751):617-8.

4. Wang D, DuBois RN. Role of prostanoids in gastrointestinal cancer. J Clin Invest. 2018;128(7):2732-42.

5. Bar-Shavit R, Maoz M, Kancharla A, Nag JK, Agranovich D, GrisaruGranovsky S, Uziely B. G protein-coupled receptors in cancer. Int J Mol Sci. 2016:17(8):1320.

6. Misawa K, Imai A, Mochizuki D, Misawa Y, Endo S, Hosokawa S, Ishikawa R, Mima M, Shinmura K, Kanazawa T, et al. Genes encoding neuropeptide receptors are epigenetic markers in patients with head and neck cancer: a site-specific analysis. Oncotarget. 2017;8(44):76318-28.

7. Wacker D, Stevens RC, Roth BL. How ligands illuminate GPCR molecular pharmacology. Cell. 2017;170(3):414-27.

8. Heng BC, Aubel D, Fussenegger M. G protein-coupled receptors revisited: therapeutic applications inspired by synthetic biology. Annu Rev Pharmacol Toxicol. 2014;54:227-49.

9. Arakaki AKS, Pan WA, Trejo J. GPCRs in cancer: protease-activated receptors, endocytic adaptors and signaling. Int J Mol Sci. 2018;19(7):1886.

10. Majumder M, Nandi P, Omar A, Ugwuagbo KC, Lala PK. EP4 as a therapeutic target for aggressive human breast cancer. Int J Mol Sci. 2018;19(4):1019.

11. Alcolea S, Anton R, Camacho M, Soler M, Alfranca A, Aviles-Jurado FX, Redondo JM, Quer M, Leon X, Vila L. Interaction between head and neck squamous cell carcinoma cells and fibroblasts in the biosynthesis of PGE2. J Lipid Res. 2012;53(4):630-42.

12. Tsuboi K, Sugimoto Y, Ichikawa A. Prostanoid receptor subtypes. Prostaglandins Other Lipid Mediat. 2002;68-69:535-56.

13. Honda T, Kabashima K. Prostanoids in allergy. Allergol Int. 2015;64(1):11-6.

14. Narumiya S, Sugimoto Y, Ushikubi F. Prostanoid receptors: structures, properties, and functions. Physiol Rev. 1999;79(4):1193-226.

15. Misawa K, Mima M, Imai A, Mochizuki D, Misawa Y, Endo S, Ishikawa R, Kanazawa T, Mineta H. The neuropeptide genes SST, TAC1, HCRT, NPY, and GAL are powerful epigenetic biomarkers in head and neck cancer: a site-specific analysis. Clin Epigenetics. 2018;10:52.

16. Mochizuki D, Misawa Y, Kawasaki H, Imai A, Endo S, Mima M, Yamada S, Nakagawa T, Kanazawa T, Misawa K. Aberrant epigenetic regulation in head and neck cancer due to distinct EZH2 overexpression and DNA hypermethylation. Int J Mol Sci. 2018;19(12):3707.

17. Imai A, Mochizuki D, Misawa Y, Nakagawa T, Endo S, Mima M, Yamada S, Kawasaki H, Kanazawa T, Misawa K. SALL2 is a novel prognostic methylation marker in patients with oral squamous carcinomas: associations with SALL1 and SALL3 methylation status. DNA Cell Biol. 2019;38(7):678-87.

18. Kune GA, Kune S, Watson LF. Colorectal cancer risk, chronic illnesses, operations, and medications: case control results from the Melbourne Colorectal Cancer Study. Cancer Res. 1988;48(15):4399-404.

19. Gurpinar E, Grizzle WE, Piazza GA. COX-independent mechanisms of cancer chemoprevention by anti-inflammatory drugs. Front Oncol. 2013;3:181. 
20. Wong RSY. Role of nonsteroidal anti-inflammatory drugs (NSAIDs) in cancer prevention and cancer promotion. Adv Pharmacol Sci. 2019;2019:3418975.

21. Nasry WHS, Rodriguez-Lecompte JC, Martin CK. Role of COX-2/PGE2 mediated inflammation in oral squamous cell carcinoma. Cancers. 2018;10(10):348.

22. Ornelas A, Zacharias-Millward N, Menter DG, Davis JS, Lichtenberger L, Hawke D, Hawk E, Vilar E, Bhattacharya P, Millward S. Beyond COX-1: the effects of aspirin on platelet biology and potential mechanisms of chemoprevention. Cancer Metastasis Rev. 2017;36(2):289-303.

23. Ricciotti E, FitzGerald GA. Prostaglandins and inflammation. Arterioscler Thromb Vasc Biol. 2011;31(5):986-1000.

24. Kumai T, Oikawa K, Aoki N, Kimura S, Harabuchi Y, Celis E, Kobayashi H. Tumor-derived TGF-beta and prostaglandin E2 attenuate anti-tumor immune responses in head and neck squamous cell carcinoma treated with EGFR inhibitor. J Transl Med. 2014;12:265.

25. Jeng JH, Wang YJ, Chiang BL, Lee PH, Chan CP, Ho YS, Wang TM, Lee JJ, Hahn LJ, Chang MC. Roles of keratinocyte inflammation in oral cancer: regulating the prostaglandin E2, interleukin- 6 and TNF-alpha production of oral epithelial cells by areca nut extract and arecoline. Carcinogenesis. 2003;24(8):1301-15.

26. Kalmar A, Peterfia B, Hollosi P, Galamb O, Spisak S, Wichmann B, Bodor A, Toth K, Patai AV, Valcz G, et al. DNA hypermethylation and decreased mRNA expression of MAL, PRIMA1, PTGDR and SFRP1 in colorectal adenoma and cancer. BMC Cancer. 2015;15:736.

27. Ooki A, Maleki Z, Tsay JJ, Goparaju C, Brait M, Turaga N, Nam HS, Rom WN, Pass HI, Sidransky D, et al. A panel of novel detection and prognostic methylated DNA markers in primary non-small cell lung cancer and serum DNA. Clin Cancer Res. 2017;23(22):7141-52.

28. Chang CC, Wang HC, Liao YP, Chen YC, Weng YC, Yu MH, Lai HC. The feasibility of detecting endometrial and ovarian cancer using DNA methylation biomarkers in cervical scrapings. J Gynecol Oncol. 2018;29(1):e17.

29. Itcho K, Oki K, Kobuke K, Yoshii Y, Ohno H, Yoneda M, Hattori N. Aberrant $G$ protein-receptor expression is associated with DNA methylation in aldosterone-producing adenoma. Mol Cell Endocrinol. 2018;461:100-4.

30. Sugino Y, Misawa A, Inoue J, Kitagawa M, Hosoi H, Sugimoto T, Imoto I, Inazawa J. Epigenetic silencing of prostaglandin E receptor 2 (PTGER2) is associated with progression of neuroblastomas. Oncogene. 2007;26(53):7401-13.

31. Tian L, Suzuki M, Nakajima T, Kubo R, Sekine Y, Shibuya K, Hiroshima K, Nakatani Y, Fujisawa T, Yoshino I. Clinical significance of aberrant methylation of prostaglandin E receptor 2 (PTGER2) in nonsmall cell lung cancer: association with prognosis, PTGER2 expression, and epidermal growth factor receptor mutation. Cancer. 2008;113(6):1396-403.

32. Farkas SA, Milutin-Gasperov N, Grce M, Nilsson TK. Genome-wide DNA methylation assay reveals novel candidate biomarker genes in cervical cancer. Epigenetics. 2013;8(11):1213-25.

33. Cebola I, Custodio J, Munoz M, Diez-Villanueva A, Pare L, Prieto P, Ausso S, Coll-Mulet L, Bosca L, Moreno V, et al. Epigenetics override pro-inflammatory PTGS transcriptomic signature towards selective hyperactivation of PGE2 in colorectal cancer. Clin Epigenetics. 2015;7:74.

34. Weiss G, Schlegel A, Kottwitz D, Konig T, Tetzner R. Validation of the SHOX2/PTGER4 DNA methylation marker panel for plasma-based discrimination between patients with malignant and nonmalignant lung disease. J Thorac Oncol. 2017;12(1):77-84.

35. Hiken JF, McDonald JI, Decker KF, Sanchez C, Hoog J, VanderKraats ND, Jung KL, Akinhanmi M, Rois LE, Ellis MJ, et al. Epigenetic activation of the prostaglandin receptor EP4 promotes resistance to endocrine therapy for breast cancer. Oncogene. 2017;36(16):2319-27.

36. Ausoni S, Boscolo-Rizzo P, Singh B, Da Mosto MC, Spinato G, Tirelli G, Spinato R, Azzarello G. Targeting cellular and molecular drivers of head and neck squamous cell carcinoma: current options and emerging perspectives. Cancer Metastasis Rev. 2016;35(3):413-26.

37. Klussmann JP. Head and neck cancer-new insights into a heterogeneous disease. Oncol Res Treat. 2017;40(6):318-9.

38. Takeshima $\mathrm{H}$, Ushijima T. Accumulation of genetic and epigenetic alterations in normal cells and cancer risk. NPJ Precis Oncol. 2019;3:7.

39. Misawa K, Imai A, Mochizuki D, Mima M, Endo S, Misawa Y, Kanazawa T, Mineta $\mathrm{H}$. Association of TET3 epigenetic inactivation with head and neck cancer. Oncotarget. 2018;9(36):24480-93.

40. Ichimura N, Shinjo K, An B, Shimizu Y, Yamao K, Ohka F, Katsushima K, Hatanaka A, Tojo M, Yamamoto E, et al. Aberrant TET1 methylation closely associated with CpG island methylator phenotype in colorectal cancer. Cancer Prev Res. 2015;8(8):702-11.

\section{Publisher's Note}

Springer Nature remains neutral with regard to jurisdictional claims in published maps and institutional affiliations.
Ready to submit your research? Choose BMC and benefit from:

- fast, convenient online submission

- thorough peer review by experienced researchers in your field

- rapid publication on acceptance

- support for research data, including large and complex data types

- gold Open Access which fosters wider collaboration and increased citations

- maximum visibility for your research: over $100 \mathrm{M}$ website views per year

At BMC, research is always in progress.

Learn more biomedcentral.com/submissions 